\title{
DETERIORO SOCIOECONÓMICO SECTORIAL DERIVADO DE UN INCREMENTO IMPOSITIVO: EL CASO DEL SECTOR VETERINARIO ESPAÑOL DE ANIMALES DE COMPAÑÍA
}

\section{SOCIO-ECONOMIC SECTORAL DETERIORATION BY A TAX INCREASE: THE CASE OF THE SPANISH VETERINARY SECTOR OF PET ANIMALS}

\author{
Óscar Gutiérrez Aragón (Universidad de Girona) ${ }^{1}$ \\ Félix Barahona Márquez (Universidad de Girona) ${ }^{2}$ \\ Gaspar Berbel Giménez (Universidad de Girona) ${ }^{3}$
}

\section{Resumen:}

El sector veterinario de animales de compañía en España se vio afectado en el año 2012 por una subida al tipo impositivo general de IVA de 13 puntos porcentuales (del 8\% al 21\%). Concretamente dicho aumento causó un notable deterioro en diversos indicadores económicos y laborales del sector en los ejercicios subsiguientes (p.e. salario medio, tasa de estabilidad en el empleo, productividad). Así mismo, en esos ejercicios provocó deterioros en la recaudación fiscal relacionada directamente con el sector. Ante este escenario, y en aplicación de las teorías de Laffer, el presente estudio propugna que un retorno al tipo de gravamen reducido de IVA, contemplado por el Gobierno en estos momentos, que debería contribuir a mejorar la viabilidad económica de las empresas y las condiciones sociolaborales de los trabajadores veterinarios.

Palabras clave: sector veterinario, IVA, subida de impuestos, Laffer, precarización.

Códigos JEL: E62, H71, L84

\begin{abstract}
:
The veterinary sector to pets in Spain was affected in 2012 by a rise to the standard VAT rate of 13 percentage points (from $8 \%$ to 21\%). Specifically, this increase caused a significant deterioration in some economic and labor indicators of the sector in subsequent years (e.g. average salary, employment stability rate, productivity). Likewise, in those years it caused deterioration in tax collection directly related to the sector. According to the theories of Laffer, a return to the reduced VAT rate, currently considered by the Government, should contribute to improve the economic viability of companies and the social and working conditions of veterinary workers.
\end{abstract}

Key words: veterinary sector, VAT, tax increase, Laffer, precarization.

JEL Codes: E62, H71, L84

\footnotetext{
1 oscar.gutierrez@mediterrani.com, Universidad de Girona.

2 felix.barahona@mediterrani.com, Universidad de Girona.

3 gaspar.berbel@mediterrani.com, Universidad de Girona.

Recibido 20 de mayo de 2019. Aceptado 12 de marzo de 2020.
} 


\section{INTRODUCCIÓN}

El Real Decreto-Ley 20/2012 ${ }^{4}$ elevó los tipos de gravamen del IVA y pasó al tipo impositivo general algunas operaciones como la prestación de servicios veterinarios a animales de compañía, que sufrió un aumento del IVA de 13 puntos porcentuales (del 8 por cien al 21 por cien). Ese incremento deterioró las cuentas de resultados de estas empresas, comprometiendo en casos su supervivencia. Así, sólo en 2014 y debido a, entre otras causas, a la subida del IVA, se cerraron en España 730 clínicas de animales de compañía (VenturaGarcía, 2016).

El sector veterinario de animales de compañía suele sufrir las consecuencias de las crisis con graves deterioros en las cifras de facturación y empleo, por lo que, en un sector ya castigado, resultaron de especial gravedad los efectos del incremento del IVA (Badiola, 2016). En este contexto, la Confederación Empresarial Veterinaria Española (CEVE) encarga la elaboración de un informe académico (Gutiérrez-Aragón y Berbel-Giménez, 2017) que documente las perniciosas consecuencias que para el sector supuso el cambio impositivo de 2012. Basándose en este informe, diversos grupos parlamentarios, a petición de la CEVE, presentaron varias enmiendas al Proyecto de Ley de Presupuestos Generales del Estado de 2018, proponiendo el retorno al tipo reducido de IVA para este sector ${ }^{5}$. Esta Ley de Presupuestos finalmente no fue aprobada por falta de apoyos en el Senado. En el Proyecto de Ley de Presupuestos Generales del Estado para $2019^{6}$, también apoyándose en el mencionado informe, se contemplaba una rebaja del IVA en servicios de veterinaria prestados a animales de compañía del 21 por cien al 10 por cien. Lamentablemente, esta ley tampoco llegó a aprobarse debido a la convocatoria de elecciones generales y la posterior disolución de las Cámaras del Parlamento, pero, en todo caso, el Gobierno resultante de esas elecciones incluyó en el Programa de Estabilidad 20192022 presentado a la Unión Europea, como medida tributaria a aplicar a partir de 2020, la reducción al 10 por cien del tipo de gravamen del IVA de los servicios veterinarios, estimando que era necesario adecuar la tributación de estos servicios a la misma que los medicamentos para uso veterinario (Ministerio de Asuntos Económicos y Transformación Digital, 2019). Por lo tanto, al redactar este artículo, la rebaja del IVA a los servicios veterinarios queda pendiente tan solo de la pertinente aprobación a través de la siguiente Ley de Presupuestos Generales del Estado o mediante otro tipo de normativa. Partiendo del mencionado informe, el presente trabajo académico analizará en profundidad la evolución de diferentes indicadores vinculados a la actividad de los servicios veterinarios a animales de compañía durante el periodo que conforman los ejercicios que preceden y siguen al cambio impositivo, con el fin de evidenciar su grave deterioro tras dicha modificación.

El tema objeto de este ensayo está estrechamente relacionado con las consecuencias de la última crisis económica, lo cual le hace disfrutar de la premisa de vigente actualidad. El objetivo principal del artículo es evidenciar el claro deterioro en diversos indicadores económicos y laborales del sector veterinario en los ejercicios subsiguientes a la aplicación del incremento impositivo del IVA. Para la consecución del propósito señalado, la metodología empleada se fundamenta en el análisis descriptivo y el tratamiento estadístico de datos secundarios de fuentes oficiales sobre la actividad del sector en España. Las conclusiones del presente trabajo defienden la propuesta de una aplicación más racional de los tipos impositivos del IVA para

\footnotetext{
${ }^{4}$ Real Decreto-Ley 20/2012, de 13 de julio, de medidas para garantizar la estabilidad presupuestaria y de fomento de la competitividad (BOE $\mathrm{n}^{\circ}$. 168, de 14 de julio de 2012, pp. 50428-50518).

${ }^{5}$ Enmiendas $n^{\circ} 3406, n^{\circ} 3417, n^{\circ} 3593, n^{\circ} 5871$ y n 5881 al articulado presentadas al Proyecto de Ley de PGE para el año 2018 (BOCG, Congreso, Serie A, núm. 20-6, de 10 de mayo de 2018, pp. 2275-2276, 2285-2286, pp. 2440-2441, pp. 4143-4144 y 4148-4149)

${ }^{6}$ Ministerio de Hacienda (2019): “Proyecto de Ley de Presupuestos Generales del Estado 2019”. Recuperado el 20 de marzo de 2019 de https://bit.ly/2uqauSD.
} 
este tipo de actividades, que, como se advertirá en posteriores epígrafes, promovería derivaciones positivas para los estamentos implicados (empresarios, trabajadores, clientes, proveedores, arcas públicas, etc.), tal y como el actual Gobierno tiene presente en sus planes impositivos a corto plazo relativos al sector.

\section{MARCO TEÓRICO Y NORMATIVO}

En el núcleo central de su obra Keynes abogaba por aumentar el gasto público, bajar los impuestos o combinar ambas medidas durante una etapa recesiva para poder salir de una crisis. En su opinión, aumentar los impuestos provoca la paradoja del descenso del consumo y la pérdida de empleo, produciendo diversos efectos negativos para las arcas públicas, como el aumento de las prestaciones por desempleo (Keynes, 1936).

Décadas después, Laffer propugna que es posible bajar los impuestos y elevar la recaudación tributaria y da a conocer su célebre Curva, la cual disfruta, desde que fue descrita por vez primera en un artículo académico por Wanninski (1978) y después ampliamente explicada y desarrollada por el propio Laffer (1981, 2004), de un amplio reconocimiento. Cuando Laffer presentó su teoría a la comunidad académica su intención era aportar un instrumento pedagógico que sirviera para advertir que no siempre el incremento de la presión impositiva debía provocar un aumento de la recaudación fiscal (Casparri y Elfenbaum, 2014). Para ello, argumentó sus tesis basándose en evidencias del pasado (Laffer, 1981). No obstante, a pesar de la gran controversia que genera su conjetura, en la mayor parte de las economías mundiales se encuentran ejemplos que la justifican, sobre todo en etapas de fuerte recesión económica (Olier, 2013), como ha sido, por ejemplo, la que comenzó en 2008.

Como toda teoría que genera profundos debates académicos, ha habido economistas y fiscalistas que la han acusado de cierta fragilidad argumental e inaplicabilidad (Analistas Económicos de Andalucía, 2008). Otros, tal y como apunta Sanz-Sanz (2017), cuestionan la posibilidad de que defina una relación inversa entre recaudación y tipos de gravamen. También hay quien estima que la curva de Laffer sólo es aplicable de forma individual a cada contribuyente (cada uno tendría su propia curva), aunque se podría inferir una curva agregada virtual de Laffer para toda la población (Sanz-Sanz, 2016). Finalmente, otros economistas defienden que las teorías de Laffer son aplicables a la totalidad del sistema impositivo (Nutahara, 2015; Rubiera et al., 2018), incluidos los impuestos sobre el consumo, pues si, mediante tributos, se sube el precio de bienes y servicios, su demanda disminuye, afectando a las rentas del capital (los empresarios facturan menos) y del empleo (se reducen los salarios medios), disminuyendo las recaudaciones de los impuestos que gravan dichas rentas.

Una de las primeras consecuencias del aumento moderado de los tipos impositivos es el incremento a corto plazo de los ingresos públicos, aunque, en muchos casos, lo es a costa de perjudicar la producción y el empleo (Boscá-Mares et al., 2017). Esta disyuntiva debería obligar a evaluar los efectos de los cambios impositivos sobre el bienestar social, sobre todo considerando que existen otras medidas (p.e. recorte del gasto público) mucho menos dañinas sobre la actividad que los aumentos de impuestos (Alesina et al., 2017). El propio ministro responsable del incremento de IVA a los servicios veterinarios, Cristobal Montoro, ha defendido en diversas ocasiones la bajada de impuestos para crecer y crear empleo (Europa Press, 2016), consiguiendo, por ejemplo, muy buenos resultados cuando rebajó los tipos del IRPF entre 2014 y 2017 (Sánchez de la Cruz, 2018).

Durante los periodos de recesión los consumidores tienden a gastar una mayor parte de sus ingresos sólo en aquellos bienes que más necesitan (Sancak et al., 2010), en los que es menos probable evitar el pago de impuestos bajando su consumo (Guedes de Oliveira y Costa, 2015). Por el contrario, la subida del IVA a los servicios veterinarios a mascotas sin duda ha 
hecho aflorar el efecto sustitución (reemplazándose bienes relativamente más caros por otros más baratos), sufriendo sus consecuencias los diversos prestadores de estos (y otros sectores afines, como el farmacéutico) (Gutiérrez-Aragón y Berbel-Giménez, 2017). En este contexto, resulta especialmente reveladora la Proposición No de Ley 162/000160 ${ }^{7}$, aprobada en el Congreso, pero vetada por el Gobierno de aquel momento, y que advertía que la subida del IVA de los servicios veterinarios suponía un grave perjuicio económico para el sector y, debido a este efecto sustitución, un incremento del riesgo sanitario para la población en general, derivado del aumento de casos o brotes de enfermedades o infecciones zoonóticas en los animales (transmisibles al ser humano en condiciones naturales).

La Ley $30 / 1985^{8}$, por la que se implanta el IVA en España, establecía exención de este impuesto a la gran mayoría de las actividades sanitarias, pero no a los servicios veterinarios, que quedaban encuadrados en el tipo general (12 por cien). La Ley 37/1992 ${ }^{9}$, actualmente vigente tras su última revisión (29-06-2017), mantiene sin exención del impuesto a los servicios veterinarios, aunque la Ley $41 / 1994^{10}$, los incluyó por vez primera en el tipo de gravamen reducido (7 por cien). La Ley 26/2009 ${ }^{11}$, incrementó el tipo impositivo reducido (8 por cien), que se ha mantenido para los servicios veterinarios a animales de compañía hasta la entrada en vigor del Real Decreto-Ley 20/2012, que los pasó al tipo general (21 por cien). Por otra parte, la Directiva 2006/112/CE ${ }^{12}$ permite a los estados miembros aplicar de forma opcional tipos de gravamen reducidos de IVA a una lista restringida de bienes y servicios, entre los que se encuentran los veterinarios (Álvarez-García, 2015), quedando esta decisión, pues, reducida al mero ejercicio de la voluntad política.

Atendiendo a la incuestionable relación entre la sanidad animal y la salud pública (MartínDíaz, 2017, Espínola, 2018) resulta paradójico el incremento impositivo a las únicas prestaciones sanitarias no exentas de tributar IVA. Además, se debe tener presente que la tenencia de animales de compañía ejerce un efecto muy beneficioso en la salud física y mental de las personas con quienes conviven (Hugues-Hernandorena et al., 2012), impactando muy positivamente en las arcas públicas, al reducirse el gasto médico y farmacéutico de estas (Headey y Grabka, 2003; Clower y Neaves, 2015; Mills et al., 2016; Wedderburn, 2017), por lo cual sería recomendable favorecer esta tenencia desde las instancias públicas, no aplicando una carga impositiva extra.

Además de los problemas de índole sanitaria, el aumento del IVA causó un notable deterioro en un gran número de indicadores económicos, financieros y laborales del sector, principalmente en los ejercicios objetos de este estudio, 2012, 2013 y 2014 (Gutiérrez-Aragón y Berbel-Giménez, 2017), disminuyendo la facturación, los beneficios y la productividad del sector, y empeorando las condiciones laborales de los trabajadores, perjudicando en mayor medida a los más jóvenes (CEVE, 2018). Si bien es cierto que, a partir de 2015, coincidiendo con la mejora económica del país, el sector veterinario experimentó una progresiva recuperación en un gran número de sus indicadores (valor añadido, facturación, producción,

\footnotetext{
${ }^{7}$ Proposición no de Ley 162/000160, sobre la rebaja del IVA en los servicios veterinarios. Boletín Oficial de las Cortes Generales, serie D, $\mathrm{n}^{\circ}$ 25, 30 de septiembre de 2016, pp. 33-35.

${ }^{8}$ Ley 30/1985, de 2 de agosto, del Impuesto sobre el Valor Añadido (BOE nº 190, de 9 de agosto de 1985, pp. 25214-25243).

${ }^{9}$ Ley 37/1992, de 28 de diciembre, del Impuesto sobre el Valor Añadido (BOE $\mathrm{n}^{\circ}$ 312, de 29 de diciembre de 1992, pp. 44247-44305).

${ }^{10}$ Ley 41/1994, de 30 de diciembre, de Presupuestos Generales del Estado para 1995 (BOE n 313 , de 31 de diciembre de 1994, pp. 39387-39456).

${ }^{11}$ Ley 26/2009, de 23 de diciembre, de Presupuestos Generales del Estado para el año 2010 (BOE n ${ }^{\circ}$ 309, de 24 de diciembre de 2009, pp. 108804-109227).

12 Directiva 2006/112/CE, del Consejo, de 28 de noviembre de 2006, relativa al sistema común del impuesto sobre el valor añadido (DOUEL n ${ }^{\circ} 347$, de 11 de diciembre de 2006).
} 
excedente bruto de explotación, compras, inversión, etc.), aquellos índices relativos a la calidad del empleo (trabajadores eventuales y no remunerados), el salario medio y la productividad por ocupado, en mayor o menor medida, siguen en valores preocupantes (Gutiérrez-Aragón, 2019).

Considerando el marco descrito, cabe referirse a la existencia de una serie de estudios macroeconómicos, que postulan que los efectos de una reducción de tipos tienden a asegurar un aumento del bienestar social y de las bases imponibles futuras, garantizando la sostenibilidad financiera presupuestaria en el largo plazo (Sanz-Sanz, 2017). Este fenómeno afectaría en su conjunto a los impuestos que gravan las rentas salariales, el capital y el consumo (Nutahara, 2015; Oudheusden, 2016; Boscá-Mares et al., 2017). Por ello, en la idea de tratar de enmendar los efectos perjudiciales que el incremento de IVA ha producido sobre el sector veterinario, principalmente en lo relativo a las condiciones sociolaborales, teniendo en cuenta que la política fiscal provoca efectos constatables a corto plazo sobre la actividad y el empleo (De Castro et al., 2010), debería abogarse por el retorno a tipos impositivos más bajos para este tipo de servicios.

\section{METODOLOGÍA}

Para alcanzar el objetivo propuesto, el presente artículo realiza un análisis descriptivo y estadístico a partir de fuentes de información secundaria, con el fin de analizar la evolución en el sector veterinario de una serie de magnitudes o variables de carácter económico como la recaudación de IVA, las cotizaciones anuales de la Seguridad Social a cargo de la empresa, el volumen de negocio, el excedente bruto explotación, el valor añadido, la inversión en activos materiales y activos intangibles, los gastos de personal, el tamaño de las empresas o el número de trabajadores eventuales y el de no remunerados, así como el comportamiento en el periodo estudiado de indicadores analizados como el salario medio, la tasa de estabilidad en el empleo o la productividad en euros.

Para poder contrastar la posible influencia del incremento de gravamen del IVA a los servicios veterinarios a animales de compañía sobre dichas variables se ha analizado la evolución de las series temporales históricas aportadas por el Instituto Nacional de Estadística (INE) en la Encuesta Anual de Servicios (CNAE-2009) relativa al CNAE 75 (actividades veterinarias) (Instituto Nacional de Estadística, 2019 a). Del INE también se han utilizado los datos sobre las series históricas de diversas variables macroeconómicas. Así mismo, se han empleado resultados procedentes de estudios previos realizados para y sobre el devenir socioeconómico del sector veterinario español (Gutiérrez-Aragón y Berbel-Giménez, 2017; Gutiérrez-Aragón et al., 2018). Para el análisis de los datos fiscales se ha utilizado los informes de recaudación de la Agencia Tributaria (2019) y para poder estimar el peso que sobre el total del sector representan los servicios a animales de compañía se han empleado los informes de la asociación empresarial Veterindustria (2008-2015). Finalmente se procedió a efectuar un análisis correlacional en base a pruebas no paramétricas, adecuadas para distribuciones reducidas de datos, que demuestran el impacto negativo del incremento del IVA sobre las empresas del sector. También se han recogido, de forma complementaria y explicativa al presente estudio, los resultados obtenidos del contraste efectuado a los datos analizados, que determinan una relación de causalidad entre el incremento de IVA y varios de los indicadores considerados. 


\section{ANÁLISIS DE RESULTADOS}

\subsection{Peso sobre el total nacional y recaudación de IVA}

Al valorar el peso del sector de las actividades veterinarias a animales de compañía en la economía nacional en el periodo analizado, los datos obtenidos arrojan una media del peso de estas actividades del 0,00704 por cien sobre el PIB nacional entre 2008 y 2015 con un máximo del 0,0087 por cien (Instituto Nacional de Estadística, 2019 b; Veterindustria, 2008-2015; Gutiérrez-Aragón y Berbel-Giménez, 2017) y una media del 0,02283 por cien sobre el total de la estimación de ingresos y gastos de los Presupuestos Generales del Estado entre 2012 a 2015 (Ministerio de Hacienda y Función Pública, 2012-2015; Veterindustria, 2008-2015). Así, a pesar de su importancia estratégica desde un punto de vista sanitario y de la salud pública, el subsector no tiene un peso excesivamente elevado sobre el PIB y los PGE.

Desde septiembre de 2012, al incrementarse el tipo de gravamen del IVA, se produce un aumento de la recaudación por este concepto, a pesar de una facturación del sector sensiblemente inferior, especialmente en el caso de animales de compañía. Así, en los tres primeros ejercicios de vigencia del cambio impositivo (2012, 2013 y 2014) se produce una disminución del 11,64 por cien del valor añadido a precios de mercado del total del sector, a la vez que la recaudación aumenta un 39,68 por cien (tabla 1). Por su parte, en esos tres ejercicios, se produce una mengua del 10,70 por cien del valor añadido a precios de mercado del subsector veterinario de animales de compañía, aumentando al tiempo su recaudación en un 134,17 por cien. Este notable incremento porcentual, sin embargo, no aporta una gran cantidad de ingresos a las arcas públicas, quedándose en esos ejercicios en una media de 8,48 millones de euros anuales, una cifra realmente pequeña, que, de acuerdo a alguna de las doctrinas apuntadas en el marco teórico del presente artículo, podría ser fácilmente compensada con una bajada del tipo de gravamen que estimulase la prestación de servicios veterinarios y la venta de fármacos asociados al aumento de prescripciones.

\begin{tabular}{|c|c|c|c|c|c|c|c|c|}
\hline \multicolumn{9}{|c|}{$\begin{array}{l}\text { TABLA 1: ESTIMACIÓN DEL INCREMENTO DE RECAUDACIÓN DE } \\
\text { IVA TRAS EL INCREMENTO IMPOSITIVO EN LOS SERVICIOS } \\
\text { VETERINARIOS A ANIMALES DE COMPAÑIIA (MILLONES DE €). }\end{array}$} \\
\hline & 2008 & 2009 & 2010 & 2011 & 2012 & 2013 & 2014 & 2015 \\
\hline $\begin{array}{l}\text { Valor añadido estimado a } \\
\text { precios de mercado } \\
\text { (animales de compañía) }\end{array}$ & 67,26 & 69,43 & 74,32 & 79,32 & 78,63 & 66,60 & 70,80 & 93,47 \\
\hline $\begin{array}{c}\text { Estimación del IVA } \\
\text { recaudado de animales de } \\
\text { compañía }\end{array}$ & 4,71 & 4,86 & 5,57 & 6,35 & 9,70 & 13,99 & 14,87 & 19,63 \\
\hline $\begin{array}{c}\text { Incremento recaudación } \\
\text { IVA vet. A animales de } \\
\text { compañía }\end{array}$ & - & - & - & - & 2,88 & 7,33 & 7,79 & 10,28 \\
\hline
\end{tabular}

Tomando como base 100 los datos del año 2008, se puede verificar como la evolución de la recaudación de IVA derivado de la prestación de servicios veterinarios a animales de compañía sufre un incremento porcentual muchísimo mayor (416,89 por cien) que el IVA total (125,58 por cien) o el IVA de todas las actividades veterinarias (199,03 por cien), advirtiendo de una evolución de la presión tributaria sobre estas actividades que quizás pueda y deba parecer un tanto injusta con el subsector (tabla 2). 
TABLA 2: EVOLUCIÓN DE LA RECAUDACIÓN DE IVA. TOTAL NACIONAL, ACTIVIDADES VETERINARIAS (DATOS EN PORCENTAJE)

\begin{tabular}{|c|c|c|c|c|c|c|c|c|}
\hline & 2008 & 2009 & 2010 & 2011 & 2012 & 2013 & 2014 & 2015 \\
\hline $\begin{array}{l}\text { Evolución de la } \\
\text { recaudación del IVA } \\
\text { total de España }\end{array}$ & 100,00 & 69,90 & 102,22 & 102,67 & 105,09 & 108,14 & 116,98 & 125,58 \\
\hline $\begin{array}{c}\text { Evolución de la } \\
\text { recaudación del IVA } \\
\text { veterinario }\end{array}$ & 100,00 & 97,74 & 103,45 & 109,89 & 125,20 & 150,15 & 153,50 & 199,03 \\
\hline $\begin{array}{l}\text { Evolución de la } \\
\text { recaudación estimada } \\
\text { del IVA veterinario de } \\
\text { animales de compañía }\end{array}$ & 100,00 & 103,22 & 118,38 & 134,78 & 205,96 & 297,06 & 315,80 & 416,89 \\
\hline
\end{tabular}

Ateniéndose a las cifras de los incrementos recaudatorios derivados del cambio del tipo impositivo del IVA a la prestación de servicios veterinarios a animales de compañía, cuando se obtiene el peso relativo de estas con respecto al PIB total o a los PGE (tabla 3), el porcentaje que suponen en relación a estas cifras macroeconómicas es insignificante, representando en el mejor de los casos un 0,0009558 por cien del PIB (media anual del 0,0008095 por cien) o un 0,0029558 por cien de los PGE (media anual del 0,0024593 por cien).

\begin{tabular}{|c|c|c|c|c|}
\hline \multicolumn{5}{|c|}{$\begin{array}{l}\text { TABLA 3: PESO DEL INCREMENTO RECAUDATORIO DE IVA POR LA } \\
\text { PRESTACIÓN DE SERVICIOS VETERINARIOS A ANIMALES DE COMPAÑA } \\
\text { CON RESPECTO AL PIB Y A LOS PGE. }\end{array}$} \\
\hline & 2012 & 2013 & 2014 & 2015 \\
\hline $\begin{array}{c}\text { Producto Interior Bruto (PIB) España } \\
\text { (millones de } € \text { ) }\end{array}$ & 1.039 .758 & 1.025 .634 & 1.037 .025 & 1.075 .639 \\
\hline $\begin{array}{l}\text { Presupuestos Generales del Estado } \\
\text { (millones de } € \text { ) }\end{array}$ & 311.777 & 345.446 & 354.626 & 347.843 \\
\hline $\begin{array}{l}\text { Incremento recaudación estimada IVA } \\
\text { veterinario a animales de compañía } \\
\text { (millones de } € \text { ) }\end{array}$ & 2,88 & 7,33 & 7,79 & 10,28 \\
\hline $\begin{array}{l}\text { Incremento recaudación IVA respecto al } \\
\text { PIB }\end{array}$ & $0,0002773 \%$ & $0,0007147 \%$ & $0,0007512 \%$ & $0,0009557 \%$ \\
\hline $\begin{array}{l}\text { Incremento recaudación IVA respecto a } \\
\text { los Presupuestos Generales del Estado }\end{array}$ & $0,0009247 \%$ & $0,0021219 \%$ & $0,0021967 \%$ & $0,0029554 \%$ \\
\hline
\end{tabular}

Además, este incremento extra de recaudación de IVA derivado del cambio impositivo a los servicios veterinarios a animales de compañía tampoco parece suponer un porcentaje ciertamente importante sobre la recaudación total del impuesto, un 0,0170490 por cien en el mejor de los casos (media anual del 0,0152205 por cien) (Ministerio de Hacienda y Función Pública, 2012-2015), un pequeño incremento recaudatorio que, sin embargo, causó graves perjuicios al sector durante el periodo analizado en el presente estudio.

Uno de estos perjuicios, asociado a la reducción de la prestación de servicios veterinarios a animales de compañía, es el descenso de la facturación de productos de sanidad y nutrición animal, que sufre un quebranto bastante grande (-9,48 por cien) justo tras producirse el cambio impositivo, a pesar de que durante la crisis económica no había tenido un mal comportamiento. 
Un análisis similar referido solamente a la facturación de medicamentos veterinarios para animales de compañía arroja un notable descenso del 10,28 por cien en 2013, a pesar de que estos continuaban gravados con el tipo reducido del IVA. Ese mismo año disminuye el 12,48 por cien del consumo de servicios veterinarios (razonablemente previsible al aumentar los precios en recesión económica) (Gutiérrez-Aragón y Berbel-Giménez, 2017). Del comportamiento conjunto de estos indicadores se puede inferir que un retorno al tipo de gravamen reducido para el IVA de las actividades veterinarias a animales de compañía no sólo beneficiaría a la facturación de las clínicas, sino también a la de los sectores afines, produciendo un aumento en la recaudación de impuestos, tanto del IVA como del Impuesto de Sociedades.

Otro de los efectos evidentes que sobre el sector produjo el incremento de 13 puntos porcentuales de gravamen de IVA en septiembre de 2012 fue que los empresarios del sector decidieron, en un gran número, compensar en ese mismo mes la subida impositiva reduciendo en una cifra similar su margen comercial, de forma que, sin variar los precios finales de venta, se produjo una rebaja efectiva de su facturación real. Evidentemente, la reducción de los márgenes resultaba tan elevada que mantener tal decisión fue inviable (inexistencia de margen comercial o resultados negativos), por lo que en los siguientes meses se pasó a repercutir a los consumidores el incremento impositivo (Instituto Nacional de Estadística, 2019 d), aumentando de forma progresiva los precios de venta al público (y, por tanto, las bases imponibles del impuesto), lo que produjo una progresiva disminución de la demanda de estos servicios.

Obviamente, en este contexto, aparecieron graves perjuicios económicos que no sólo los sufrieron las empresas veterinarias, sino también los clientes (incremento de precios), los proveedores (reducción de ventas), los trabajadores (deterioro de las condiciones salariales y laborales) y las propias arcas públicas (que, aunque en estas actividades aumentaron los ingresos por IVA, disminuyeron las recaudaciones del impuesto de sociedades, al disminuir los beneficios de explotación, y de seguridad social y de IRPF, en virtud de una menor retribución salarial a los trabajadores). Así, en lo referente a la recaudación de las cotizaciones a la Seguridad Social a cargo de las empresas, tomando como base el año anterior a la entrada en vigor del cambio impositivo (2011), se advierte un deterioro de los ingresos públicos por este concepto de más de cuatro millones de euros en sólo tres ejercicios, de 2012 a 2014 (tabla 4), derivado de la disminución salarial de los profesionales del sector, el aumento de la precariedad (mayor parcialidad contractual y pérdida de empleo) o de ambas circunstancias a un mismo tiempo.

\begin{tabular}{|c|c|c|c|c|}
\hline \multicolumn{5}{|c|}{$\begin{array}{l}\text { TABLA 4: DISMINUCIÓN DE COTIZACIONES ANUALES DE LA } \\
\text { SEGURIDAD SOCIAL A CARGO DE LA EMPRESA (MILES DE EUROS). } \\
\text { ACTIVIDADES VETERINARIAS. }\end{array}$} \\
\hline & 2011 & 2012 & 2013 & 2014 \\
\hline $\begin{array}{l}\text { Cotizaciones a la Seguridad Social a cargo de } \\
\text { la empresa }\end{array}$ & 35.649 & 33.790 & 35.243 & 33.824 \\
\hline $\begin{array}{l}\text { Disminución ingresos por cotizaciones } \\
\text { anuales con respecto al año } 2011\end{array}$ & - & -1.859 & -406 & -1.825 \\
\hline $\begin{array}{l}\text { Disminución acumulada ingresos por } \\
\text { cotizaciones con respecto al año } 2011\end{array}$ & - & -1.859 & -2.265 & -4.090 \\
\hline
\end{tabular}




\subsection{Evolución salarial y de los indicadores del empleo}

En el periodo objeto de análisis el total de sueldos y salarios percibidos en el sector disminuyó un 6,30 por cien, de 149,02 a 139,63 millones de euros, y los gastos totales de personal se vieron reducidos en un 6,26 por cien, de 186,44 a 174,78 millones de euros (Instituto Nacional de Estadística, 2019 c). Además, la disminución de los salarios y el aumento de la eventualidad provocó un aumento extraordinario del autoempleo, por lo que al final del año 2014 el 58,66 por cien de las empresas veterinarias eran unipersonales $(8,11$ por cien más que en 2010). Las pequeñas empresas, de 2 a 4 personas, disminuyeron un 14,87 por cien, al pasar gran parte de estas al primero de los grupos. El número de las empresas de 20 a 99 personas se redujo en un 23,08 por cien (Instituto Nacional de Estadística, 2019c). La tendencia a la fragmentación suele ser indicativo de precarización del empleo.

El salario medio del sector en los peores años de la crisis había seguido una tendencia descendente similar a la de otros sectores, pero la decisión de incrementar la carga impositiva a estos servicios provocó una considerable recaída (tabla 5), acumulándose al final de 2015 una pérdida salarial del 13,31 por cien con respecto a 2009 (2.036 € menos en términos absolutos). Este indicador, a pesar de la mejoría del sector a partir de 2015, seguía en 2017 (último ejercicio del que ofrece datos el INE) bastante lejos de recuperarse (la pérdida salarial era aún del 10,43 por cien, $1.545 €$ menos en términos absolutos) (Gutiérrez-Aragón, 2019). Obviamente, los empresarios del sector trataron de compensar parte de la pérdida de rentas de capital con una sucesión de progresivas disminuciones de las rentas del trabajo de sus empleados.

\begin{tabular}{|cccccccc|}
\hline \multicolumn{7}{|c|}{ TABLA 5: SALARIO MEDIO. ACTIVIDADES VETERINARIAS } \\
\hline & $\mathbf{2 0 0 9}$ & $\mathbf{2 0 1 0}$ & $\mathbf{2 0 1 1}$ & $\mathbf{2 0 1 2}$ & $\mathbf{2 0 1 3}$ & $\mathbf{2 0 1 4}$ & $\mathbf{2 0 1 5}$ \\
\hline Salario medio (euros) & 15.294 & 15.472 & 15.239 & 14.864 & 14.285 & 13.363 & 13.258 \\
\hline Variación anual (euros) & 178 & -233 & -375 & -579 & -922 & -105 \\
\hline $\begin{array}{c}\text { Variación anual } \\
\text { (porcentaje) }\end{array}$ & $1,16 \%$ & $-1,51 \%$ & $-2,46 \%$ & $-3,90 \%$ & $-6,45 \%$ & $-0,78 \%$ \\
\hline $\begin{array}{c}\text { Variación acumulada del } \\
\text { salario medio (euros) }\end{array}$ & $\mathbf{1 7 8}$ & $-\mathbf{5 5}$ & $\mathbf{- 4 3 0}$ & $\mathbf{- 1 . 0 0 9}$ & $\mathbf{- 1 . 9 3 1}$ & $\mathbf{- 2 . 0 3 6}$ \\
\hline $\begin{array}{c}\text { Variación acumulada del } \\
\text { salario medio (porcentaje) }\end{array}$ & $\mathbf{1 , 1 6} \%$ & $\mathbf{- 0 , 3 6} \%$ & $\mathbf{- 2 , 8 1} \%$ & $\mathbf{- 6 , 6 0} \%$ & $\mathbf{- 1 2 , 6 3 \%}$ & $\mathbf{- 1 3 , 3 1} \%$ \\
\hline Fuente: Instituto Nacional de Estadística (2019c). & & & & & \\
\hline
\end{tabular}

Al comparar la evolución del salario medio anual de las actividades veterinarias con los de diversas actividades y ocupaciones se puede verificar que al final de 2015, ya en plena recuperación macroeconómica y con la mayoría de los sectores con valores similares a los de 2009, se encontraba por debajo de los de actividades con cualificación y requerimiento de formación similares (actividades científicas o sanitarias), e incluso también de otras de mucha menor cualificación (actividades administrativas, restauración y comercio, hostelería, peones, etc.), representando el 86,69 por cien del que había en 2009, en un periodo en el que el salario medio global se incrementó en un 2,64 por cien (tabla 6). 


\begin{tabular}{|c|c|c|c|}
\hline \multicolumn{4}{|c|}{$\begin{array}{l}\text { TABLA 6: EVOLUCIÓN DE LOS SALARIOS MEDIOS POR GRUPOS } \\
\text { DE OCUPACIÓN. BASE 100: AÑO } 2009 .\end{array}$} \\
\hline Actividad / Ocupación & 2009 & 2015 & $\begin{array}{c}\text { Variación } \\
\text { (base 100: 2009) }\end{array}$ \\
\hline Salario medio global & 22.511 & 23.106 & $102,64 \%$ \\
\hline Actividades profesionales, científicas y técnicas & 25.517 & 26.347 & $103,25 \%$ \\
\hline Actividades sanitarias y de servicios sociales & 25.623 & 25.824 & $100,78 \%$ \\
\hline $\begin{array}{c}\text { Peones (agricultura, pesca, construcción, industrias } \\
\text { manufactureras y transportes) }\end{array}$ & 15.343 & 16.209 & $105,64 \%$ \\
\hline Actividades administrativas y servicios auxiliares & 15.855 & 15.949 & $100,59 \%$ \\
\hline Trabajadores de los servicios de restauración y comercio & 14.651 & 14.699 & $100,33 \%$ \\
\hline Hostelería & 13.997 & 13.977 & $99,86 \%$ \\
\hline Actividades veterinarias & 15.294 & 13.258 & $86,69 \%$ \\
\hline Instituto Nacional de Estadística (2019c. 2019e) & & & \\
\hline
\end{tabular}

De hecho, el coste salarial ordinario (en euros/mes) de los trabajadores veterinarios en 2015, pocos ejercicios después del cambio impositivo, era de $997 € /$ mes, en el puesto 75 de las 79 actividades de la CNAE, sólo por encima de actividades como la jardinería, servicios de comidas, peluquería o actividades de entretenimiento (Instituto Nacional de Estadística, 2015; Jiménez, 2015). Esta cifra suponía menos de la mitad del salario medio de las actividades sanitarias (2.132,90 €/mes).

El alcance del aumento de la precariedad laboral en el sector se puede constatar examinando la evolución del número de trabajadores eventuales del sector y el de trabajadores no remunerados (personas que trabajan en la empresa regularmente sin percibir salario: propietarios, familiares y gerentes autónomos). Ambos indicadores, que habían descendido en el periodo 2008-2011, soportaron grandes incrementos en el bienio 2013-2014, justo tras la subida impositiva, llegando a alcanzar en el periodo 2008-2014 un 36,97 por cien más de trabajadores eventuales y 12,97 por cien más de no remunerados (tabla 7). A partir de ese momento, el deterioro de estos indicadores ha seguido creciendo hasta alcanzar en 2017 un 59,27 por cien más de trabajadores eventuales y un 25,48 por cien más de no remunerados respecto a 2008 (Instituto Nacional de Estadística 2019c; Gutiérrez-Aragón, 2019). 
TABLA 7: NÚMERO DE TRABAJADORES EVENTUALES Y NÚMERO DE TRABAJADORES NO REMUNERADOS. ACTIVIDADES VETERINARIAS.

\begin{tabular}{cccccccc}
\hline & $\mathbf{2 0 0 8}$ & $\mathbf{2 0 0 9}$ & $\mathbf{2 0 1 0}$ & $\mathbf{2 0 1 1}$ & $\mathbf{2 0 1 2}$ & $\mathbf{2 0 1 3}$ & $\mathbf{2 0 1 4}$ \\
\hline $\begin{array}{c}\text { Número de trabajadores } \\
\text { eventuales }\end{array}$ & 1.694 & 1.862 & 1.574 & 1.607 & 1.762 & 2.361 & 2.285 \\
\hline Variación anual & - & $9,92 \%$ & $-15,47 \%$ & $2,10 \%$ & $9,65 \%$ & $34,00 \%$ & $-3,22 \%$ \\
\hline Variación acumulada & - & $\mathbf{9 , 9 2} \%$ & $\mathbf{- 5 , 5 5} \%$ & $-\mathbf{3 , 4 5} \%$ & $\mathbf{6 , 1 9} \%$ & $\mathbf{4 0 , 1 9} \%$ & $\mathbf{3 6 , 9 7 \%}$ \\
\hline $\begin{array}{c}\text { Número de trabajadores } \\
\text { no remunerados }\end{array}$ & 7.951 & 8.088 & 8.144 & 7.674 & 8.359 & 8.413 & 8.981 \\
\hline Variación anual & - & $1,72 \%$ & $0,69 \%$ & $-5,77 \%$ & $8,93 \%$ & $0,65 \%$ & $6,75 \%$ \\
\hline Variación acumulada & - & $\mathbf{1 , 7 2} \%$ & $\mathbf{2 , 4 2} \%$ & $-\mathbf{3 , 3 6} \%$ & $\mathbf{5 , 5 7} \%$ & $\mathbf{6 , 2 2} \%$ & $\mathbf{1 2 , 9 7 \%}$ \\
\hline F, 2, & \\
\hline
\end{tabular}

Fuente: Instituto Nacional de Estadística (2019c).

Es de resaltar que el número de trabajadores no remunerados había tenido un descenso importante (5,77 por cien) en el ejercicio 2011. El hecho de que coincida una caída general del número de desempleados a nivel nacional con el ascenso posterior de los trabajadores no remunerados en el sector veterinario sólo puede tener una causa de tipo artificial que afecte exclusivamente a este sector. Esta sólo puede ser en ese periodo el incremento del tipo de gravamen del IVA.

Por su lado, de 2008 a 2014 el número de trabajadores remunerados aumentó, alcanzando un 110,65 por cien respecto al inicio. Si se compara esta variable con la evolución del salario medio, que descendió a un 92,52 por cien (sobre todo a partir del incremento impositivo), y con los gastos en personal (prácticamente iguales) (Instituto Nacional de Estadística, 2019c; Gutiérrez-Aragón y Berbel-Giménez, 2017), debe concluirse que este contexto sólo puede producirse si existe un mayor número de empleados a tiempo parcial (lo cual, unido a salarios más reducidos, revela un aumento de la precariedad laboral). De hecho, la tasa de estabilidad en el empleo (proporción de trabajadores con contrato fijo sobre el número de remunerados totales) había alcanzado su techo en el periodo en 2011 (84,30 por cien), justo el último año completo con gravamen reducido de IVA. A partir de entonces, los sucesivos decrementos la hicieron caer hasta el 79,40 por cien en 2014. El descenso de 2012 y 2013, tras el cambio impositivo, fue de un 7,22 por cien. En 2017, con un 80,83 por cien, seguía bastante lejos de sus mejores valores (Instituto Nacional de Estadística, 2019c).

\subsection{Valor añadido a precios de mercado}

Al examinar la evolución en el periodo analizado del valor añadido a precios de mercado que genera el sector en su conjunto (indicador que excluye el IVA para su cálculo), es posible comprobar como el año 2014 había sufrido un deterioro del 15,78 por cien con respecto a las cifras de 2008, lo cual suponía aproximadamente una disminución de 52 millones de euros (tabla 8). El examen de la evolución de esta variable es sumamente interesante para los contenidos del presente artículo, pues sobre ella se ha de calcular el impuesto de valor añadido devengado por la administración tributaria en cada periodo. A pesar de los descensos moderados que venía sufriendo esta cifra desde el comienzo de la crisis económica, es precisamente en los ejercicios 2012 y 2013 (el de implantación del cambio de gravamen y el 
inmediatamente posterior) cuando sufre sus más serias mermas, de un 4,81 por cien y un 8,28 por cien, respectivamente.

\begin{tabular}{|c|c|c|c|c|c|c|c|}
\hline \multicolumn{8}{|c|}{$\begin{array}{l}\text { TABLA 8: EVOLUCIÓN DEL VALOR AÑADIDO A PRECIOS DE MERCADO. } \\
\text { ACTIVIDADES VETERINARIAS }\end{array}$} \\
\hline & 2008 & 2009 & 2010 & 2011 & 2012 & 2013 & 2014 \\
\hline $\begin{array}{l}\text { Valor añadido a precios de } \\
\text { mercado (miles de euros) }\end{array}$ & 346.317 & 338.487 & 334.389 & 332.994 & 316.977 & 290.745 & 294.228 \\
\hline $\begin{array}{c}\text { Variación anual del valor } \\
\text { añadido a precios de mercado } \\
\text { (miles de euros) }\end{array}$ & - & -7.830 & -4.098 & -1.395 & -16.017 & -26.232 & 3.483 \\
\hline $\begin{array}{c}\text { Variación anual del valor } \\
\text { añadido a precios de mercado }\end{array}$ & - & $-2,26 \%$ & $-1,21 \%$ & $-0,42 \%$ & $-4,81 \%$ & $-8,28 \%$ & $1,20 \%$ \\
\hline $\begin{array}{l}\text { Variación acumulada del valor } \\
\text { añadido a precios de mercado } \\
\text { (miles de euros) }\end{array}$ & - & -7.830 & -11.928 & -13.323 & -29.340 & -55.572 & -52.089 \\
\hline $\begin{array}{l}\text { Variación acumulada del valor } \\
\text { añadido a precios de mercado }\end{array}$ & - & $-2,26 \%$ & $-3,47 \%$ & $-3,89 \%$ & $-8,70 \%$ & $-16,97 \%$ & $-15,78 \%$ \\
\hline
\end{tabular}

Si se relaciona la evolución del valor añadido a precios de mercado con la de los gastos de personal, se puede observar como a medida que la primera variable desciende, la segunda aumenta (tabla 9). Resulta especialmente significativo el comportamiento de ambos indicadores en 2013, tras el cambio impositivo, pues siendo un ejercicio de aparente recuperación general, coincide el menor valor añadido generado (es el de menor facturación de la serie) con un aumento en el gasto de personal, lo cual sólo resulta posible con un incremento notable de la eventualidad (un 34 por cien en 2013).

\begin{tabular}{|c|c|c|c|c|c|c|c|}
\hline \multicolumn{8}{|c|}{$\begin{array}{l}\text { TABLA 9: COMPARATIVA DE LA EVOLUCIÓN DEL VALOR AÑADIDO } \\
\text { A PRECIO DE MERCADO Y LOS GASTOS DE PERSONAL DE LAS } \\
\text { ACTIVIDADES VETERINARIAS. BASE 100: AÑO } 2008 .\end{array}$} \\
\hline & 2008 & 2009 & 2010 & 2011 & 2012 & 2013 & 2014 \\
\hline $\begin{array}{c}\text { Evolución del } \\
\text { valor añadido a } \\
\text { precios de } \\
\text { mercado }\end{array}$ & $100,00 \%$ & $97,74 \%$ & $96,56 \%$ & $96,15 \%$ & $91,53 \%$ & $83,95 \%$ & $84,96 \%$ \\
\hline $\begin{array}{c}\text { Evolución gastos } \\
\text { de personal }\end{array}$ & $100,00 \%$ & $102,90 \%$ & $104,38 \%$ & $107,19 \%$ & $103,04 \%$ & $104,81 \%$ & $100,49 \%$ \\
\hline
\end{tabular}

\subsection{Productividad en euros por persona ocupada}

Uno de los indicadores que resultó seriamente afectado fue la productividad en euros por persona ocupada. Tomando como base 2008, tras unos descensos moderados, habituales en recesión, a partir de 2012 las caídas de productividad se disparan hasta el 22,23 por cien acumulado en 2014 (4.445 euros menos por trabajador que en 2008) (tabla 10). Obviamente, en este comportamiento, entre otras causas, influyó en gran medida la bajada de los precios de 
los servicios veterinarios tras la subida impositiva. Por otra parte, resulta lógico que, si los ingresos por facturación disminuyen y aumenta el número de empleados ocupados, la productividad por empleado disminuya. Los graves deterioros de la productividad del sector veterinario en 2012 (-7,39 por cien) y 2013 (-11,40 por cien) son también efecto del incremento del IVA, al provocarse una considerable reducción de los ingresos en un sector que hasta esa coyuntura era uno de los que había resistido mejor la crisis económica (Gutiérrez-Aragón et al., 2018). En 2017, a pesar de la recuperación económica del sector, la productividad todavía era un 4,43 por cien menor que en 2008 (887 € en términos absolutos).

\begin{tabular}{|lrrrrrrr}
\hline \multicolumn{7}{|c|}{ TABLA 10: EVOLUCIÓN DE LA PRODUCTIVIDAD (€/PERSONA OCUPADA). } \\
ACTIVIDADES VETERINARIAS. \\
\hline $\mathbf{2 0 0 8}$ & $\mathbf{2 0 0 9}$ & $\mathbf{2 0 1 0}$ & $\mathbf{2 0 1 1}$ & $\mathbf{2 0 1 2}$ & $\mathbf{2 0 1 3}$ & $\mathbf{2 0 1 4}$ \\
\hline Productividad (euros) & 19.998 & 19.935 & 19.739 & 19.285 & 17.859 & 15.823 & 15.553 \\
\hline $\begin{array}{l}\text { Variación anual de la } \\
\text { productividad (euros) }\end{array}$ & -63 & -196 & -454 & -1.426 & -2.036 & -270 \\
\hline $\begin{array}{c}\text { Variación anual de la } \\
\text { productividad }\end{array}$ & $-0,32 \%$ & $-0,98 \%$ & $-2,30 \%$ & $-7,39 \%$ & $-11,40 \%$ & $-1,71 \%$ \\
\hline $\begin{array}{l}\text { Variación acumulada de } \\
\text { la productividad (euros) }\end{array}$ & -63 & -259 & -713 & -2.139 & -4.175 & -4.445 \\
\hline $\begin{array}{c}\text { Variación acumulada de } \\
\text { la productividad }\end{array}$ & $-0,32 \%$ & $-1,30 \%$ & $-3,57 \%$ & $-10,70 \%$ & $-20,88 \%$ & $-22,23 \%$ \\
\hline
\end{tabular}

Fuente: Instituto Nacional de Estadística (2019c).

Relacionando la evolución del valor añadido a precios de mercado y la productividad con el número de empleados ocupados en el sector (tabla 11), es posible verificar la relación inversa entre los dos primeros indicadores, que disminuyeron progresivamente (hasta un 84,96 por cien y 77,77 por cien en 2014 con respecto a 2008, respectivamente), con la ocupación, que aumentó (110,61 por cien), prueba del crecimiento de la precarización laboral en el sector. En 2012 y 2013, tras el cambio impositivo, fue cuando más se incrementó esta desviación.

\begin{tabular}{|c|c|c|c|c|c|c|c|}
\hline \multicolumn{8}{|c|}{$\begin{array}{l}\text { TABLA 11: COMPARATIVA DE LA EVOLUCIÓN DEL VALOR AÑADIDO } \\
\text { A PRECIO DE MERCADO, PRODUCTIVIDAD Y NÚMERO DE } \\
\text { OCUPADOS. ACTIVIDADES VETERINARIAS. BASE 100: AÑO } 2008 .\end{array}$} \\
\hline & 2008 & 2009 & 2010 & 2011 & 2012 & 2013 & 2014 \\
\hline $\begin{array}{l}\text { Evolución del valor } \\
\text { añadido a precios } \\
\text { de mercado }\end{array}$ & $100,00 \%$ & $97,74 \%$ & 96,56 \% & $96,15 \%$ & $91,53 \%$ & $83,95 \%$ & $84,96 \%$ \\
\hline $\begin{array}{l}\text { Evolución de la } \\
\text { productividad }\end{array}$ & $100,00 \%$ & $99,68 \%$ & $98,70 \%$ & $96,43 \%$ & $89,30 \%$ & $79,12 \%$ & $77,77 \%$ \\
\hline $\begin{array}{l}\begin{array}{l}\text { Evolución de } \\
\text { empleados } \\
\text { ocupados }\end{array} \\
\end{array}$ & $100,00 \%$ & $99,36 \%$ & $99,43 \%$ & $100,08 \%$ & $102,62 \%$ & $106,30 \%$ & $110,61 \%$ \\
\hline
\end{tabular}




\subsection{Volumen de negocio de las empresas}

El volumen de negocio de las empresas del sector fue una de las variables que en el periodo estudiado más deterioro sufrió. En ello influyeron tanto la crisis como la decisión de incrementar el gravamen del IVA, siendo precisamente el año 2013 el que coincidió con una menor facturación de la serie (tabla 12). Considerando que este indicador recoge los importes netos facturados por las empresas incluyendo todos los impuestos con excepción del IVA repercutido a los clientes, parece lógico que sea precisamente durante el periodo en el que los veterinarios deciden compensar la subida del IVA con una reducción de sus márgenes (bajando los precios antes de impuestos) cuando se presenten las cifras más bajas de volumen de negocio (683 millones de euros en 2013). En 2014 se había perdido un 8,19 por cien del volumen de negocio que había en 2008 (casi 63 millones de euros de reducción).

\begin{tabular}{|cccccccc|}
\hline \multicolumn{7}{|c|}{ TABLA 12: EVOLUCIÓN DEL VOLUMEN DE NEGOCIO. ACTIVIDADES } \\
VETERINARIAS. \\
\hline & $\mathbf{2 0 0 8}$ & $\mathbf{2 0 0 9}$ & $\mathbf{2 0 1 0}$ & $\mathbf{2 0 1 1}$ & $\mathbf{2 0 1 2}$ & $\mathbf{2 0 1 3}$ & $\mathbf{2 0 1 4}$ \\
\hline $\begin{array}{c}\text { Volumen de negocio (miles de } \\
\text { euros) }\end{array}$ & 770.696 & 754.607 & 756.078 & 730.796 & 720.785 & 683.194 & 707.988 \\
\hline $\begin{array}{c}\text { Variación anual del volumen } \\
\text { de negocio (miles de euros) }\end{array}$ & - & -16.089 & 1.471 & -25.282 & -10.011 & -37.591 & 24.794 \\
\hline $\begin{array}{c}\text { Variación anual del volumen } \\
\text { de negocio }\end{array}$ & - & $-2,09 \%$ & $0,19 \%$ & $-3,34 \%$ & $-1,37 \%$ & $-5,22 \%$ & $3,63 \%$ \\
\hline $\begin{array}{c}\text { Variación acumulada del } \\
\text { volumen de negocio (miles de } \\
\text { euros) }\end{array}$ & - & $\mathbf{- 1 6 . 0 8 9}$ & $\mathbf{- 1 4 . 6 1 8}$ & $\mathbf{- 3 9 . 9 0 0}$ & $\mathbf{- 4 9 . 9 1 1}$ & $\mathbf{- 8 7 . 5 0 2}$ & $\mathbf{- 6 2 . 7 0 8}$ \\
\hline $\begin{array}{c}\text { Variación acumulada del } \\
\text { volumen de negocio }\end{array}$ & - & $\mathbf{- 2 , 0 9} \%$ & $\mathbf{- 1 , 8 9} \%$ & $\mathbf{- 5 , 2 4} \%$ & $\mathbf{- 6 , 6 1} \%$ & $\mathbf{- 1 1 , 8 2} \%$ & $\mathbf{- 8 , 1 9} \%$ \\
\hline Fuente: Instituto Nacional de Estadística (2019c). & & & & & \\
\hline
\end{tabular}

\subsection{Excedente bruto de explotación}

Un indicador financiero muy relevante al tratar de analizar el comportamiento de un sector empresarial durante un periodo determinado es el excedente bruto de explotación (tabla 13), cuya evolución determina si un sector presenta señales de fortaleza o de deterioro. Esta variable recoge el remanente del valor añadido una vez remunerado el factor trabajo. La evolución de este indicador en el sector ha sido relativamente mala durante la crisis, pero sobre todo lo fue en el ejercicio que siguió al cambio impositivo del IVA, cayendo un 20,92 por cien. En 2014 comienza a recuperarse, tras el incremento de los precios de venta y la reducción de salarios. La mengua acumulada entre 2008 y 2014 fue del 29,43 por cien (49,5 millones de euros). 
TABLA 13: EVOLUCIÓN DEL EXCEDENTE BRUTO DE EXPLOTACIÓN. ACTIVIDADES VETERINARIAS.

\begin{tabular}{|c|c|c|c|c|c|c|c|}
\hline & 2008 & 2009 & 2010 & 2011 & 2012 & 2013 & 2014 \\
\hline $\begin{array}{l}\text { Excedente bruto de } \\
\text { explotación (miles de euros) }\end{array}$ & 174.675 & 166.308 & 160.581 & 150.030 & 140.281 & 110.936 & 125.131 \\
\hline $\begin{array}{l}\text { Variación anual del } \\
\text { excedente bruto de } \\
\text { explotación (miles de euros) }\end{array}$ & - & -8.367 & -5.727 & -10.551 & -9.749 & -29.345 & 14.195 \\
\hline $\begin{array}{l}\text { Variación anual del } \\
\text { excedente bruto de } \\
\text { explotación }\end{array}$ & - & $-4,79 \%$ & $-3,44 \%$ & $-6,57 \%$ & $-6,50 \%$ & $-20,92 \%$ & $12,80 \%$ \\
\hline $\begin{array}{l}\text { Variación acumulada del } \\
\text { excedente bruto de } \\
\text { explotación (miles de euros) }\end{array}$ & - & -8.367 & -14.094 & -24.645 & -34.394 & -63.739 & -49.544 \\
\hline $\begin{array}{l}\text { Variación acumulada del } \\
\text { excedente bruto de } \\
\text { explotación } \\
\end{array}$ & - & $-4,79 \%$ & $-8,23 \%$ & $-14,80 \%$ & $-21,30 \%$ & $-42,22 \%$ & $-29,43 \%$ \\
\hline
\end{tabular}

\subsection{Análisis estadístico de variables e indicadores}

Por último, se han calculado las correlaciones existentes entre el incremento sufrido de IVA y diversas variables e indicadores utilizados en la elaboración del presente artículo (tabla 14). A tenor de los resultados, existe una relación negativa y significativa entre ellas, lo que advierte que el aumento del IVA repercute negativamente sobre varios indicadores asociados a la productividad, inversiones, dimensión de las empresas y estabilidad del empleo, como son la productividad en euros por ocupado, el porcentaje de clínicas grandes (20 a 99 trabajadores), el excedente bruto explotación, el valor añadido al coste de los factores y la tasa de estabilidad en el empleo.

\begin{tabular}{|c|c|c|c|}
\hline \multicolumn{4}{|c|}{$\begin{array}{l}\text { TABLA 14: ANÁLISIS ESTADÍSTICO DE INDICADOR } \\
\text { PEARSON Y SIGNIFICACIÓN. }\end{array}$} \\
\hline VARIABLE & CORRELACIÓN DE PEARSON & SIGNIFICACIÓN & $\mathbf{n}$ \\
\hline Tasa de estabilidad en el empleo & $-0,717$ & 0,070 & 7 \\
\hline Productividad (euros) & $-0,833^{*}$ & 0,010 & 8 \\
\hline $\begin{array}{l}\text { Porcentaje de clínicas grandes de } 20 \\
\text { a } 99 \text { trabajadores }\end{array}$ & $-0,708 *$ & 0,049 & 8 \\
\hline Volumen de negocio (miles euros) & $-0,002$ & 0,996 & 8 \\
\hline Valor añadido a precios de mercado & $-0,313$ & 0,450 & 8 \\
\hline Excedente bruto explotación & $-0,976^{* *}$ & 0,000 & 7 \\
\hline $\begin{array}{l}\text { Inversión en activos materiales } \\
\text { (miles euros) }\end{array}$ & $-0,514$ & 0,238 & 7 \\
\hline $\begin{array}{l}\text { Inversión activos intangibles (miles } \\
\text { euros) }\end{array}$ & $-0,644$ & 0,118 & 7 \\
\hline $\begin{array}{l}\text { Valor añadido al coste de los } \\
\text { factores }\end{array}$ & $-0,990 * *$ & 0,000 & 7 \\
\hline
\end{tabular}




\section{CONCLUSIONES}

A la luz de los resultados analizados, el incremento del tipo de gravamen de IVA en los servicios veterinarios generado tras la promulgación del Real Decreto-Ley 20/2012 resultó perjudicial para los intereses económicos del tejido empresarial del sector en los ejercicios 2012, 2013 y 2014, constatándose el grave deterioro de diferentes variables e indicadores económicos, financieros y sociales relacionados con las cifras agregadas del sector veterinario en este periodo. En concreto, se evidenciaron importantes decrementos en volumen de negocio, valor añadido, porcentaje de empresas grandes, cotizaciones de la Seguridad Social a cargo de las empresas y excedente bruto de explotación, que, con la mejora macroeconómica del país, se han recuperado de forma notable a partir de 2015 (Gutiérrez-Aragón, 2019). Sin embargo, otros, como el salario medio, la tasa de estabilidad en el empleo y la productividad por persona ocupada seguían en 2017, en mayor o menor medida, en cifras alejadas de las previas al incremento impositivo. Además, las condiciones laborales de los trabajadores veterinarios que se deterioraron en ese periodo no han vuelto a restablecerse (incremento de trabajadores eventuales y no remunerados, del número de empresas veterinarias unipersonales, y, en general, de la precariedad en el empleo). Por otro lado, también fueron afectados intereses de diversos stakeholders del sector, como los clientes (alza de los precios) o los proveedores (disminución de ventas). Al mismo tiempo, mientras se producía un deterioro de la salud pública, las arcas del Estado, a pesar de que aumentaron levemente la recaudación del IVA de estas actividades entre 2012 y 2014, redujeron las procedentes de las cotizaciones a la Seguridad Social del sector, la del Impuesto de Sociedades y del IRPF de los trabajadores (Gutiérrez-Aragón y Berbel-Giménez, 2017).

Una de las principales limitaciones al alcance de la investigación deriva de la gran complicación existente para determinar qué parte del deterioro sufrido por alguna de las variables e indicadores del sector veterinario correspondía al efecto de la crisis económica y qué parte provenía del incremento del IVA en el periodo inmediatamente subsiguiente al cambio fiscal. En cualquier caso, al comparar las variaciones sufridas por determinados índices nacionales con sus correspondientes al sector veterinario entre 2012 y 2014 se puede observar, por ejemplo, que el salario medio de los veterinarios cayó un 10,10 por cien en ese periodo, mientras el salario medio nacional aumentaba un 0,58 por cien. Del mismo modo, la productividad por trabajador decreció un 12,91 por cien en el sector veterinario, mientras ascendía un 1,15 por cien en el total del país. Por su parte, la caída del valor de la producción de las actividades veterinarias fue 6,46 veces más acentuada que la del total nacional (-1,68 por cien frente a -0,26 por cien) (Instituto Nacional de Estadística, 2019b, 2019c, 2019e). En los tres casos el diferencial existente entre las cifras del sector con la media de estas cifras a nivel nacional resulta significativo. Aun así, hay que admitir el gran inconveniente que supone trabajar con series temporales tan cortas, pues dificulta sobremanera un tratamiento estadístico concluyente.

De hecho, esta ha sido otra de las significativas limitaciones del estudio, pues, al trabajar con una muestra de registros muy reducida, no resulta posible aplicar de una forma eficiente técnicas de reducción de información como el análisis factorial, por lo que hubo que optar por el análisis correlacional en base a pruebas no paramétricas, más robustas y adecuadas para distribuciones reducidas de datos donde las distribuciones no siguen un patrón normal. De todos modos, al aplicar a estos registros dichas pruebas, considerando la variación del IVA como variable independiente, se puso de manifiesto una relación de causalidad entre el incremento de IVA y el nivel de ocupados, la estabilidad en el empleo y la productividad (Gutiérrez Aragón et al., 2018). 
Muchos economistas consideran que las teorías de Laffer sobre los efectos de los incrementos o decrementos en las tasas impositivas son aplicables al conjunto del sistema impositivo que afecta a un sector o a un país. Así, si el factor fundamental para incrementar el IVA a la prestación de servicios veterinarios a animales de compañía fue el aumento de los ingresos públicos, cabe preguntarse si la recaudación de una cifra tan exigua, una media de 8,48 millones de euros anuales en el periodo analizado en este trabajo (media del 0,0152205 por cien de la recaudación total de IVA), justifica de algún modo los graves perjuicios originados al sector expuestos a lo largo de este artículo.

Resulta extremadamente complejo establecer cuál sería el comportamiento de los ingresos públicos provenientes de la fiscalidad aplicada a este sector en el momento en que se produzca el retorno al tipo reducido de IVA (pendiente tan solo de aprobación en el momento de redactar este artículo, debido al amplio consenso parlamentario existente en este sentido), aunque parece claro que se incrementaría la recaudación de las cotizaciones de la Seguridad Social (al mejorar el empleo y los salarios), del IRPF (al aumentar los salarios medios) y del Impuesto de Sociedades (al crecer los beneficios) (Gutiérrez-Aragón y Berbel-Giménez, 2017). En cualquier caso, esta reducción impositiva, al permitir trasladar una parte sustancial del gasto de los clientes de los servicios veterinarios empleado actualmente en pagar el impuesto hacia la remuneración del trabajo o del capital, debería contribuir a mejorar la viabilidad económica de las empresas y, sobre todo, las condiciones sociolaborales de los trabajadores, evitando además el consecuente deterioro de la salud pública que se suele producir cuando el sector pasa por dificultades económicas.

\section{BIBLIOGRAFÍA}

Agencia Tributaria (2019): “Informes anuales de Recaudación Tributaria”. Recuperado el 2 de marzo de 2019 de http://cort.as/6OVa.

Alesina, A., Barbiero, O., Favero, C., Giavazzi, F. \& M. Paradisi (2017): “The Effects of Fiscal Consolidations: Theory and Evidence”, NBER Working Paper, $\mathrm{n}^{\circ}$ 23385, pp. 1-65.

Álvarez-García, S. (2015): Análisis del IVA aplicable en la prestación de servicios veterinarios, CEVE / Universidad de Oviedo.

Analistas Económicos de Andalucía (2008): “La curva de Laffer: Estimación para la economía española”, Documento de Análisis, nº 2, pp. 1-9.

Badiola, J.J. (2016): “Toma de posesión del nuevo presidente del Consejo de Colegios Veterinarios de Castilla y León”, citado en EFE, 2016, “Badiola alerta de la precariedad laboral de los profesionales veterinarios”, La Vanguardia, 11-06-2016.

Boscá-Mares, J.E., Doménech-Vilariño, R. y Ferri-Carreres, J. (2017): "Estructura fiscal, crecimiento económico y bienestar en España”, Papeles de Economía Española, n ${ }^{\circ} 154$, pp. 250-264.

Casparri, M.T. y Elfenbaum M. (2014): "La curva de Laffer y el impuesto inflacionario", Revista de Investigación en Modelos Matemáticos aplicados a la Gestión y la Economía, año 1, vol. 1, pp. 89-97.

CEVE (Confederación Empresarial Veterinaria Española) (2018): "Las clínicas veterinarias aportarían 5 millones de euros más cada año al Estado si se les aplicara el IVA reducido”, Revista Argos, no 197, pp. 6.

Clower T.L. \& Neaves, T.T. (2015): The Health Care Cost Savings of Pet Ownership, The Human Animal Bond Research Initiative (HABRI) Foundation. Washington.

De Castro, F., Gordo, E. y Pérez, J.J. (2010): "La política fiscal discrecional en tiempos de crisis”, Presupuesto y Gasto Público, vol. 2, n 59, pp. 83-102. 
Espínola, V. (2018): "Reducir el IVA veterinario aumentaría aún más los ingresos del Estado”, Animal's Health, El Diario de la Salud Animal. Recuperado el 5 de febrero de 2020 de https://bit.ly/3711qAT.

Europa Press (2016): "Montoro achaca el menor paro a las bajadas de impuestos y critica las amenazas de subida”, 02/06/2016. Recuperado el 28 de marzo de 2019 de http://cort.as/$7 \mathrm{k}-2$.

Guedes de Oliveira, F. \& Costa, L. (2015): "The VAT Laffer Curve and the Business Cycle in the EU27: An Empirical Approach”, Economic Issues Journal Articles, vol. 20, n 2, pp. 29-43.

Gutiérrez-Aragón, Ó. y Berbel-Giménez, G. (2017): Informe sobre la situación socioeconómica y laboral del sector veterinario de animales de compañía en España. El deterioro de la profesión tras el incremento del tipo de gravamen de IVA y propuesta de soluciones, Confederación Empresarial Veterinaria Española (adscrita a CEOE), pp. 1-74. Madrid.

Gutiérrez-Aragón, Ó., Barahona-Márquez, F. y Berbel-Giménez, G. (2018): "Efectos del incremento del tipo de gravamen del IVA en 2012 sobre el empleo y las condiciones laborales en el sector veterinario en España. Influencia en la profundización de la brecha laboral de género”, Estudios de Economía Aplicada, vol. 36, nº 3, pp. 921-944.

Gutiérrez-Aragón, Ó. (2019): Informe sobre la situación socioeconómica y laboral del sector veterinario en España. Año 2018, Confederación Empresarial Veterinaria Española (adscrita a CEOE), pp. 1-53. Madrid.

Headey, B. \& Grabka, M. (2003): "Pet Ownership is Good for your Health and Saves Public Expenditure Too: Australian and German longitudinal evidence”, Australian Social Monitor, no 4, pp. 93-99.

Hugues-Hernandorena, B., Álvarez-Álvarez, A. M., Castelo-Elias-Calles, L., Ledón-Llanes, L., Mendoza-Trujillo, M. y Domínguez-Alonso, E. (2012): “Tenencia de animales de compañía. Posibles beneficios para las personas con endocrinopatías y enfermedades metabólicas”, REDVET, Revista Electrónica de Veterinaria, vol. 13, n 6, pp. 1-13.

Instituto Nacional de Estadística (2015): "Coste salarial ordinario en el primer trimestre de 2015, en euros al mes”. En El País (2015): “Los sueldos en España, por sectores”, 22/06/2015.

Instituto Nacional de Estadística (2019a): “Clasificación Nacional de Actividades Económicas. CNAE”. Recuperado el 1 de marzo de 2019 de http://cort.as/-7k-5.

Instituto Nacional de Estadística (2019b): "Series históricas: Producto interior bruto a precios de mercado; Actividad, ocupación y paro; Cuentas Económicas Nacionales”. Recuperado el 1 de marzo de 2019 de http://www.ine.es/inebmenu/indice.htm.

Instituto Nacional de Estadística (2019c): "Estadística Estructural de Empresas: Sector Servicios 2015. Encuesta anual de servicios Series Históricas 2008-2014”. Recuperado el 28 de marzo de 2019 de http://cort.as/-7k-D.

Instituto Nacional de Estadística (2019d): "Índice de Precios de Consumo a Impuestos Constantes (2015=100). España, General y subíndices (09.3.4/5)”. Recuperado el 4 de marzo de 2019 de http://www.ine.es/jaxiT3/Tabla.htm?t=13892\&L=0.

Instituto Nacional de Estadística (2019e): "Encuestas de estructura salarial”. Recuperado el 17 de marzo de 2019 de http://cort.as/-7k-G.

Jiménez, M. (2015): “¿Cuáles son los sectores con mejores sueldos? ¿Y los más precarios?”, El País, 22-06-2015.

Keynes, J.M. (1936): The general theory of employment, interest and money, Palgrave Macmillan. Londres.

Laffer, A.B. (1981): “Government, exactions and revenue deficiencies”, Cato Journal, Vol. 1, $\mathrm{n}^{\circ} 1$. 
Laffer, A.B. (2004): “The Laffer Curve: Past, Present, and Future”, Executive Summary Backgrounder, The Heritage Foundation, $n^{\circ}$ 1765, pp. 1-16.

Martín-Díaz, J. (2017): Utilidad de los bacteriófagos y otros indicadores de contaminación fecal en muestras ambientales sólidas, Tesis Doctoral, Departamento de Genética, Microbiología y Estadística. Universidad de Barcelona.

Mills, D.S., Hall, S., Dolling, L., Bristow, K. \& Fuller, T. (2016): Companion animal economics: the economic impact of companion animals in the UK, Centre for Agriculture and Biosciences International (CABI). Wallingford, Oxfordshire.

Ministerio de Asuntos Económicos y Transformación Digital (2019): “Actualización del Programa de estabilidad 2019. Reino de España. 2019-2022”. Recuperado el 5 de febrero de 2020 de https://bit.ly/31AVvBy

Ministerio de Hacienda y Función Pública (2012-2015): "Presupuestos Generales del Estado". Recuperado el 1 de febrero de 2019 de http://cort.as/-7k-K.

Nutahara, K. (2015): “Laffer curves in Japan”, Journal of the Japanese and International Economies, vol. 36, pp. 56-72.

Olier, E. (2013): "La curva de Laffer: la relación entre impuestos e ingresos del Estado", El Economista, 31/05/2013. Recuperado el 22 de marzo de 2019 de http://cort.as/-7k

Oudheusden, P. (2016): "Fiscal policy reforms and dynamic Laffer effects", International Tax and Public Finance, n. 23, pp. 490-521.

Rubiera, F., Varela-Candamio, L. y López-García, E. (2018): “La curva de Laffer: aplicación al caso español con perspectiva espacial”, XL International Conference on Regional Science: Financing and the Role of Regions and Towns in Economic Recovery. Zaragoza, 20 de noviembre de 2014.

Sancak, C., Velloso, R. \& Xing, J. (2010): “Tax Revenue Response to Business Cycle”, IMF Working Paper WP/10/71, International Monetary Fund. Washington DC.

Sánchez de la Cruz, D. (2018): “Curva de Laffer: Montoro bajó por fin el IRPF... y recaudó un 6\% más”, Libre Mercado, 17/03/2018. Recuperado el 22 de marzo de 2019 de http://cort.as/-7k-N.

Sanz-Sanz, J.F. (2016): "The Laffer curve in schedular multi-rate income taxes with nongenuine allowances: An application to Spain”, Economic Modelling, vol. 55, pp. 42-56.

Sanz-Sanz, J.F. (2017): "La curva de Laffer: ¿mito o realidad? Discusión, modelización y evidencias en el IRPF español”, Papeles de Economía Española, n 154, pp. 179-197.

Wanniski, J. (1978): “Taxes, revenues, and the Laffer curve”, The Public Interest, $\mathrm{n}^{\circ}$ 50, pp. 316.

Ventura-García, J. (2016): "El Congreso de los Diputados debatirá el 4 de octubre una proposición no de ley para bajar el IVA veterinario”, Argos Portal Veterinaria, 26/09/2016. Recuperado el 28 de marzo de 2019 de http://cort.as/-7k-Q.

Wedderburn, P. (2017), "Pets can save money for the health service in Ireland", Wicklow People, 18/02/2017. Recuperado el 30 de enero de 2019 de http://cort.as/-7k-Z.

Veterindustria (2008-2015), Memorias anuales, Asociación Empresarial Española de la Industria de Sanidad y Nutrición Animal. Madrid. 